\title{
Midazolam Inhibits the Apoptosis of Astrocytes Induced by Oxygen Glucose Deprivation via Targeting JAK2-STAT3 Signaling Pathway
}

\author{
Li Liua Qi You ${ }^{\mathrm{b}}$ Yingfeng Tuc Quanyi Lia Lihong Zhenga Xuan Lia Jing Gua \\ Guonian Wanga
}

aDepartment of Anesthesiology, the Third Hospital of Harbin Medical University, Harbin, bepartment of Gastrointestinal Surgery, the Third Hospital of Harbin Medical University, Harbin, 'Department of Cardiology, the Fourth Hospital of Harbin Medical University, Harbin, China

\section{Key Words}

Midazolam • JAK2/STAT3 • Astrocytes $•$ Apoptosis

\begin{abstract}
Background: There is an increasing interest in the role of astrocytes contributing to the intrinsic bioremediation of ischemic brain injury. The purpose of this study was to disclose the effects and mechanism of midazolam (MDZ) on the proliferation and apoptosis of astrocytes under oxygen glucose deprivation (OGD) condition. Methods: The astrocytes were assigned randomly into four groups: control group, OGD group, OGD+MDZ group, and OGD+MDZ+IL-6 group. The astrocytes were treated with MDZ at dose of $10 \mu \mathrm{mol} / \mathrm{L}$ in OGD+MDZ group. And in OGD+MDZ+IL-6 group, the astrocytes were treated with MDZ at dose of $10 \mu \mathrm{mol} / \mathrm{L}$ and IL-6 at dose of $50 \mathrm{ng} / \mathrm{mL}$. MTT assay was used to assess cell proliferation, and cell apoptosis was analyzed by TUNEL apoptosis assay kit and flow cytometry. Furthermore, the expression of JAK2, p-JAK2, STAT3, p-STAT3, BCl-2, Bax and Caspase-3 proteins were determined by western blotting assay. Results: Astrocytes proliferation was decreased obviously in OGD group, while MDZ could increase astrocytes proliferation under OGD condition. Moreover, OGD could induce apoptosis in astrocytes and MDZ could play an anti-apoptotic role. However, IL-6, a JAK2 activator, could attenuate cell proliferation and anti-apoptotic effects of MDZ in astrocytes. In addition, the expression of $\mathrm{BCl}-2$ protein in MDZ group increased markedly, while the JAK2/ STAT3 signal proteins, Bax and Caspase- 3 proteins decreased relative to OGD group. But IL-6 could counteract the anti-apoptotic effects of MDZ. Conclusion: Midazolam has protective effects on the proliferation and apoptosis of astrocytes via JAK2/STAT3 signal pathway in vitro. We firstly disclose the beneficial roles of midazolam in astrocytes under ischemic condition, which may be a rational treatment selection for ischemic cerebral protection.
\end{abstract}




\section{Introduction}

As one of the most common chronic diseases in the world, cerebral ischemia is still a major global health challenge, which has high morbidity and mortality [1]. Growing body of evidence discloses that the normal function of the central nervous system depends on the adequate maintenance of the neuronal microenviroment [2]. This requires regulation of the extracellular $\mathrm{pH}$, osmolarity, and ionic composition, and prevention of accumulation of neurotransmitters within the synaptic space. In addition, there should be a continuous supply of nutrient content for local increase in blood and oxidative neuronal metabolism to meet the demands of the active neuronal populations. Increasing evidence shows that in normal brain, astrocytes and neurons form a complex, symbiotic relationship for both maintenance of neuronal function as well as support of brain metabolism [3, 4].The past several decades have given rise to many important discoveries and novel insights into the role of astrocytes in normal brain function and disease. In brief, astrocyte functions include buffering the extracellular space, providing substrates to neurons, interchanging glutamate and glutamine for synaptic transmission with neurons, and facilitating access to blood vessels [5-7].Glycogen has been implicated indirectly in neuron function, for example, buffering low glucose conditions. Astrocytes can also store reserve energy as glycogen, whereas neurons cannot. As astrocytes are responsible for neuronal metabolic and trophic support, astrocyte dysfunction will compromise postischemic neuronal survival. In recent years, in order to disclose the potential molecular mechanisms and roles, more researches have been focused on the effects of astrocytes in ischemic brain injury $[8,9]$.

Apoptosis, also called programmed cell death, is a pathway of cell death distinguished by such features as chromatin and cytoplasmic condensation and nuclear fragmentation, which has been implicated in several disorders of the central nervous system including ischemic brain injury [10], Alzheimer's disease [11], and multiple sclerosis [12]. But the focus of most these reported studies has been on neuronal apoptosis. Astrocytes, which comprise nearly $70 \%$ of the cells within the cerebral cortex, have been well recognized as supportive cells for neurons. However, the relationship between neurons protection and ischemia-induced apoptosis of astrocytes has not been fully elucidated.

Hypoxic or hypoxic-ischemic pre-conditioning has been shown to induce the overexpression of hypoxia inducible factor- $1 \alpha$ (HIF-1 $\alpha)$ and its downstream target survival genes such as erythropoietin (EPO) and vascular endothelial growth factor (VEGF) in rodents. And the effects of HIF-1 $\alpha$, EPO and VEGF on cell survival likely involve the expression of JAK2/STAT3 signal pathway, which plays a crucial role in regulation of inflammatory reaction [13], the growth and differentiation of cells [14], and cells apoptosis [15]. IL-6 is a member of the neuropoietin family of cytokines which includes leukemia inhibitory factor, ciliary neurotrophic factor, cardiotrophin oncostatin M, and IL-11 [16, 17]. And activated IL-6 receptor recruits JAK1, JAK2, and Tyk2 tyrosine kinases which can phosphorylate STAT1 and STAT3 $[18,19]$. Midazolam is a potent benzodiazepine derivative with powerful hypnotic, sedative, anxiolytic, amnestic, anticonvulsant and muscle-relaxant properties by modulating the $\mathrm{GABA}_{\mathrm{A}}$ receptor in the central nervous system [20] . Recent study has demonstrated that astrocytic activation, maturation, and differentiation are involved in the expression of central type benzodiazepine receptors coupled to $\mathrm{GABA}_{\mathrm{A}}$ receptors in astrocytes. Furthermore, Hiroki et al. has demonstrated that midazolam inhibits IL-1b-induced IL- 6 release through the JAK/STAT3 pathway suppression in C6 glioma cells [21]. However, the cytoprotective effect of midazolam on brain astrocytes has not been fully disclosed.

The aims of the present study are to determine the role of midazolam involved in cytoprotective effect on brain astrocytes and further disclose the potential molecular mechanism. We firstly performed experiments in vitro to demonstrate the pro-proliferation role of astrocytes under oxygen glucose deprivation (OGD) condition. The data generated from experiments in vitro revealed that midazolam inhibits OGD-induced apoptosis by suppressing the JAK2/STAT3 signaling pathway in brain astrocytes. In conclusion, this study 


\section{Cellular Physiology $\quad$ Cell Physiol Biochem 2015;35:126-136 \\ \begin{tabular}{ll|l} 
DOI: 10.1159/000369681 & \\
and Biochemistry & $\begin{array}{l}\text { P 2015 S. Karger AG, Basel } \\
\text { www.karger.com/cpb }\end{array}$ \\
\cline { 2 - 3 }
\end{tabular} \\ Liu et al.: Midazolam Inhibits Astrocytes Apoptosis via Targeting JAK2/STAT3 Signaling Pathway}

indicated that manipulation of JAK2/STAT3 signal pathway by midazolam represents a promising therapeutic strategy for cytoprotective effect on brain astrocytes.

\section{Materials and Methods}

\section{Preparation of Cerebral Astrocytic Cultures}

All animal experimental procedures were carried out using protocols approved by the Experimental Animal Ethic Committee of Harbin Medical University, China. Sprague-Dawley (SD) rats aged less than $48 \mathrm{~h}$ were supplied by the Medical Experimental Animal Center of Harbin Medical University. The rat astrocytes dissociation procedure was similar to that described previously by Schaefer et al. [22]. Briefly, meningesfree cortices were cut into small cubes $\left(1 \mathrm{~mm}^{3}\right)$ and digested with trypsin for $10 \mathrm{~min}$ at $37^{\circ} \mathrm{C}$. After being mechanically dissociated, the cell suspension was sieved through nylon filters. Cultures were maintained in DMEM/F12 with $10 \%$ fetal bovine serum (FBS), $100 \mathrm{U} / \mathrm{ml}$ penicillin, and $100 \mathrm{mg} / \mathrm{ml}$ streptomycin. All cultures were incubated at $37^{\circ} \mathrm{C}$ with $5 \% / 95 \% \mathrm{CO}_{2} /$ air $(\mathrm{v} / \mathrm{v})$ and $95 \%$ humidity. Cultures became confluent in around 2 weeks, which were used for this study.

Oxygen and Glucose Deprivation (OGD)

On the day of the experiment, the culture medium was removed, the cells were washed with warm phosphate-buffered saline (PBS), and then the experimental medium was added. For experiments at normal culture conditions (21\% oxygen and with glucose), the experiment medium was DMEM (glucose concentration at $5.5 \mathrm{mM}$ ). To achieve OGD, a technique was similar to that described by Iraburu and Li [23]. Briefly, the OGD experimental medium (DMEM without glucose) was previously gassed with nitrogen for 30 min and then added to cell culture wells, which had been washed three times with PBS. OGD was induced by incubating cells in ahumidified airtight chamber (Billups-Rothberg Inc., Del Mar, CA) equipped with an air lock and continuously flushed with $95 \% \mathrm{~N}_{2} / 5 \% \mathrm{CO}_{2}$ for 15 min under $37^{\circ} \mathrm{C}$. The airtight chamber was then sealed and kept in a $37^{\circ} \mathrm{C}$ incubator for $2 \mathrm{~h}, 4 \mathrm{~h}, 6 \mathrm{~h}$, or $12 \mathrm{~h}$. The oxygen concentration was below $0.2 \%$, as monitored by an oxygen analyzer (Sable Systems, Las Vegas, NV). Vehicle (DMSO), Midazolam (10 $\mu$ mol/L, Jiangsu Nhwa Pharmaceutical Limited Corporation, China) or IL-6 (50 ng/mL, B\&D Technologies Inc., USA) was added $2 \mathrm{~h}$ before the start of OGD, and the DMSO concentration in the experimental medium was less than $0.1 \%$.

\section{Cell proliferation assay evaluation using MTT test}

Primary culture astrocytes were plated in 96-well plates and treated with OGD, OGD+MDZ, or OGD+MDZ+IL- 6 respectively. After the treatment period, the serum-free medium was removed, and then the cells were cultured with regular culture medium for another $48 \mathrm{~h}$. To monitor cell survival, astrocytes were incubated for $4 \mathrm{~h}$ with $0.5 \mathrm{mg} / \mathrm{mL}$ of MTT (Sigma), and resuspended in $150 \mu \mathrm{L}$ of DMSO (Sigma). Absorbance was recorded at $490 \mathrm{~nm}$ using an Easy Reader 340 AT (SLT-Lab Instruments). Results are presented as percentage of survival taking the control as $100 \%$ survival. Experiments were performed in triplicate.

\section{TUNEL analysis}

Apoptosis-induced DNA fragmentation was determined using the transferase mediated deoxyuridine triphosphate(dUTP)-digoxigenin nick-end labeling (TUNEL) assay [24]. The astrocytes from different groups were fixed with $4 \%(\mathrm{w} / \mathrm{v}$ ) paraformaldehyde and processed by using a commercial kit (Roche) in accordance with the manufacturer's instructions. TUNEL staining was done using the in situ cell death detection kit (Roche) and the nuclei were stained with DAPI for $10 \mathrm{~min}$. The data were expressed as a percentage of the area of TUNEL-positive cells in 10 random fields. The astrocytes apoptosis was determined by Image Pro Plus software.

\section{Cell apoptosis analysis by FACS}

FACS analysis was performed as previously described [25]. Cell apoptosis was determined using an annexin V-FITC apoptosis detection kit (BD Biosciences). Briefly, $2.0 \times 10^{5}$ cells were resuspended in 0.5 $\mathrm{ml}$ of binding buffer and incubated with annexin V-FITC and propidiumiodide for $10 \mathrm{~min}$ in the dark at room temperature. A FACScan flow cytometer (BD Biosciences) equipped with a FITC signal detector FL1 
(excitation $488 \mathrm{~nm}$, green) and a phycoerythrin emission signal detector FL3 (excitation $585 \mathrm{~nm}$, red) was used to analyze cellular apoptosis. We performed three independent tests. The results were calculated using the Cell Quest ${ }^{\mathrm{TM}}$ Pro software (BD Biosciences) and expressed as the percentage of apoptotic cells of the total cells.

\section{Western-blotting analysis}

Total protein samples were extracted from the cultured astrocytes for protein immunoblotting, with the procedures essentially the same as described in details elsewhere. Equal amounts of protein $(100 \mu \mathrm{g})$ were fractionated by SDS-PAGE (8\%-15\% polyacrylamide gels) and blotted to PVDF membrane. Membranes were blocked for $2 \mathrm{~h}$ in 5\% non-fat milk in Tris-buffered saline with Tween (TBST), then probed overnight at $4{ }^{\circ} \mathrm{C}$ with the following primary antibodies respectively: JAK2 (1:500 dilution, Cell Signaling), p-JAK2 (1:500 dilution, Cell Signaling), STAT3 (1:200 dilution, Abcam), p-STAT3 (and 1:200 dilution, Abcam), Bcl-2 (1:500 dilution, Abcam), Bax (1:500 dilution, Abcam) and Caspase-3 (1:500 dilution, Santa Cruz). Following incubation with the primary antibodies, membranes were incubated with secondary antibody: Alexa Fluor ${ }^{\circledR} 800$ goat anti-mouse or anti-rabbit IgG (Invitrogen) diluted at 1:8000 at room temperature for $1 \mathrm{~h}$. Western blot bands were captured by using the Odyssey Infrared Imaging System (LI-COR Biosciences) and quantified with Odyssey v1.2 software (LI-COR Biosciences), using $\beta$-actin as an internal control. Western blotting experiments were repeated four times unless otherwise stated.

\section{Statistical Analysis}

All quantitative data are expressed as the mean \pm standard error of the mean (SEM). Statistical analysis was performed using the Student's $t$ test for comparisons of two groups and using one-way ANOVA for multi-group comparisons. Significance was set at $P<0.05$.

\section{Results}

Midazolam reduces OGD-induced damage in astrocytes

In order to induce damage in astrocytes, the cells were cultured in a humidified airtight chamber equipped with an air lock and continuously flushed with $95 \% \mathrm{~N}_{2} / 5 \% \mathrm{CO}_{2}$ for $15 \mathrm{~min}$ under $37^{\circ} \mathrm{C}$. The airtight chamber was then sealed and kept in a $37^{\circ} \mathrm{C}$ incubator for $2 \mathrm{~h}, 4 \mathrm{~h}$, $6 \mathrm{~h}$, and $12 \mathrm{~h}$. The astrocytes viability determined by MTT analysis was reduced nearly by 40 percent in cells upon OGD for $6 \mathrm{~h}$ (Fig. 1A). Next, we investigated the possible cytoprotective effect of midazolam on OGD-induced damages in astrocytes. The cells were pretreated with $1,3,10,15$ or $30 \mu \mathrm{M}$ midazolam for $2 \mathrm{~h}$ and then cultured under OGD condition for $6 \mathrm{~h}$. The result showed that pretreatment with midazolam reduced OGD-induced cell death of astrocytes in a dose dependent manner, with a remarkable cytoprotective effect at $10 \mu \mathrm{M}$ midazolam (Fig. 1B).

In addition, we further evaluated whether IL-6, could attenuate the role of midazolam in astrocytes survival under OGD condition for $6 \mathrm{~h}$. The astrocytes viability determined by MTT analysis was increased significantly in cells pretreated with midazolam $(P<0.05)$ (Fig. 1C), but not in cells co-treated with the midazolam and IL-6 ( $P>0.05)$ (Fig. 1C).

\section{Midazolam can inhibit OGD-induced astrocytes apoptosis}

To evaluate the extent of apoptosis in astrocytes, apoptotic cells were stained using the TUNEL method. The number of apoptotic-positive cells was counted in a high-power field ( $\times 200$ magnification). A notable increase of apoptotic-positive cells was observed in the OGD treatment group, compared with the control group (Fig. 2A). However, MDZ could markedly play anti-apoptotic role in astrocytes under OGD condition. Data of apoptotic indexes of the four groups were shown as follows: control group $(5.03 \pm 0.44) \%$, OGD group $(29.15 \pm 1.40) \%$, OGD+MDZ group $(14.34 \pm 0.77) \%$ and OGD+MDZ+IL-6 group $(26.23 \pm 1.70)$ $\%$ (Fig. 2A and $2 \mathrm{~B}$ ).

Employing FITC-conjugated annexin $V$ to label cell surface inverted phosphatidyl serine on apoptotic cells, we found that pre-treatment of midazolam, the apoptotic rate of OGD- 


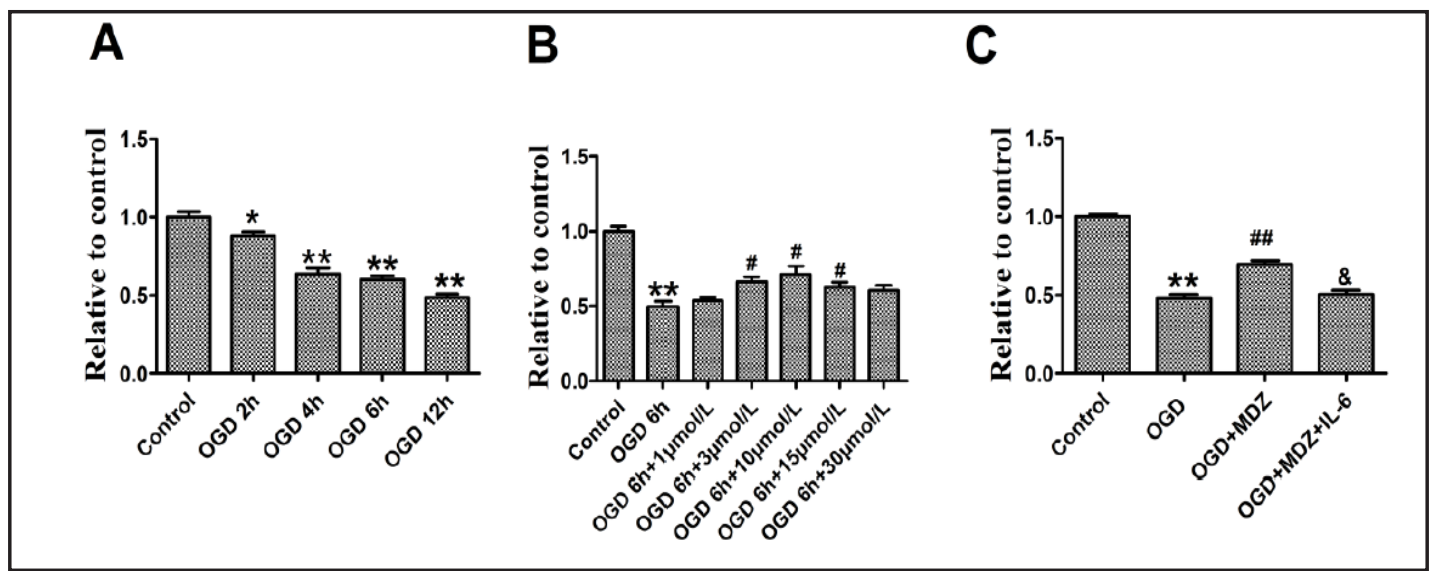

Fig. 1. The effect of midazolam on the proliferation of astrocytes in vitro. (A) Astrocytes were treated with OGD for $2 \mathrm{~h}, 4 \mathrm{~h}, 6 \mathrm{~h}$ or $12 \mathrm{~h}$, respectively. Cell viability was detected by MTT assays. Data are expressed as mean \pm SEM, $n=3,{ }^{* *} \mathrm{P}<0.01$ vs control; (B) Astrocytes were pretreated with $1,3,10,15$ or $30 \mu$ midazolam for $2 \mathrm{~h}$ and then cultured under OGD condition for $6 \mathrm{~h}$. Cell viability was also detected by MTT assays. Data are expressed as mean $\pm \mathrm{SEM}, \mathrm{n}=3,{ }^{* *} \mathrm{P}<0.01$ vs control; $\mathrm{P}<0.05$ vs OGD group;(C) MTT assay of the proliferation of astrocytes in control group, OGD group, OGD+MDZ group and OGD+MDZ+IL-6 group. Data are expressed as mean $\pm \mathrm{SEM}, \mathrm{n}=3,{ }^{* *} \mathrm{P}<0.01$ vs control; ${ }^{\# \#} \mathrm{P}<0.01$ vs $\mathrm{OGD}$ group, ${ }^{\&} \mathrm{P}<0.05$ vs $\mathrm{OGD}+\mathrm{MDZ}$ group.

Fig. 2. The effect of midazolam on apoptosis of astrocytes under OGD condition. Quantification of apoptotic astrocytes was determined and shown in the diagram. (A) Apoptotic astrocytes were determined by TUNEL staining and visualized at $200 \times$ magnification. Green color is TUNEL staining representing apoptotic cell; blue color is the cell nucleus stained by DAPI. (B) The number of apoptotic cells was significantly increased in cells treated with OGD than in control group, while the midazolam could attenuate the effect of OGD. Data are expressed as mean \pm SEM, $\mathrm{n}=10,{ }^{* *} \mathrm{P}<0.01$ vs control, ${ }^{*} \mathrm{P}<0.05$ vs OGD group, ${ }^{\& \&} \mathrm{P}<0.01$ vs $\mathrm{OGD}+\mathrm{MDZ}$ group.

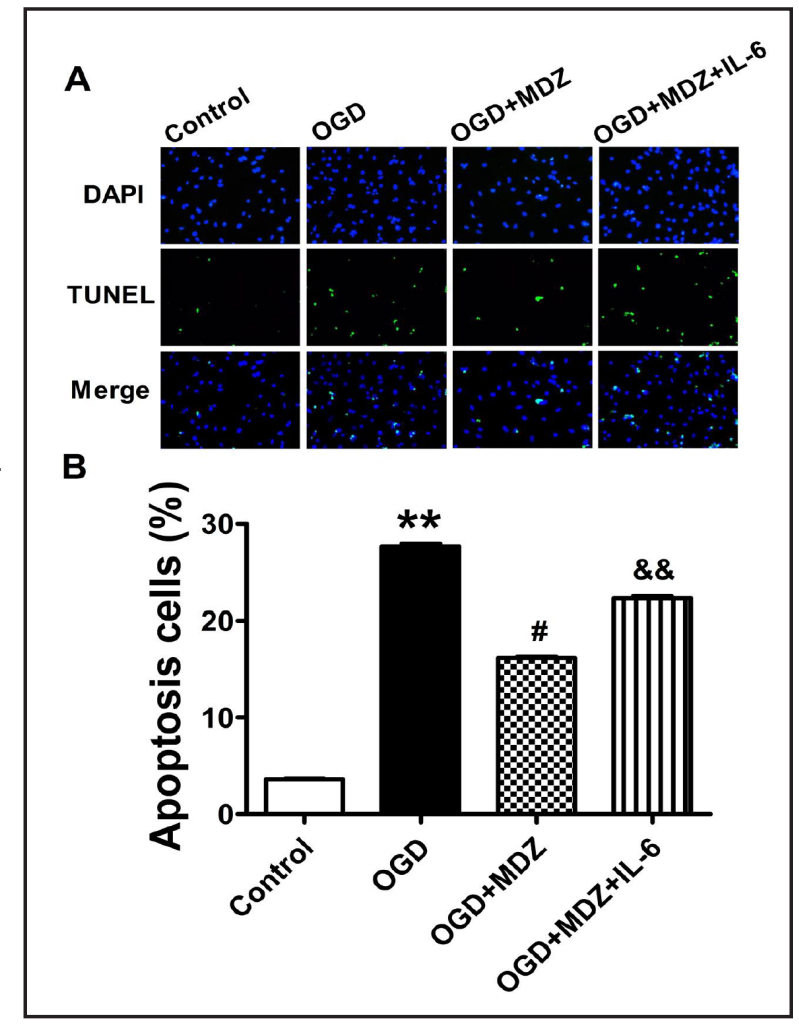

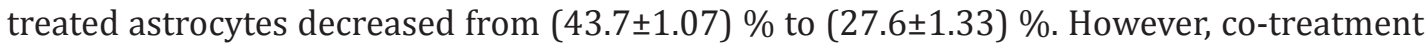
of midazolam and IL- 6 did not markedly change the apoptotic rate of astrocytes induced by OGD treatment relative to OGD group (Fig. 3A and 3B). These data suggested that midazolam 
A
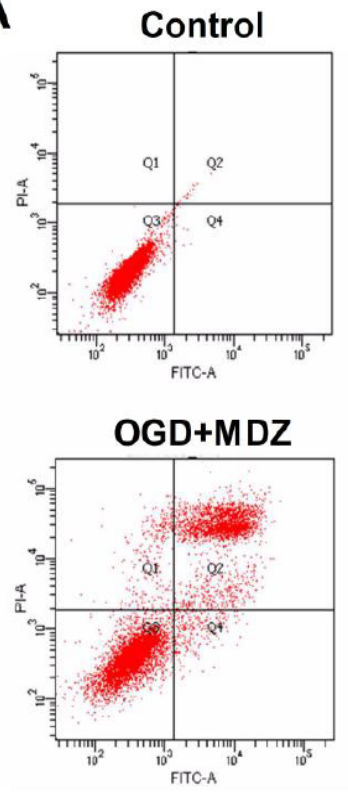

OGD

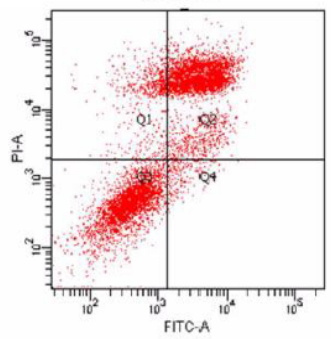

OGD+MDZ+IL-6

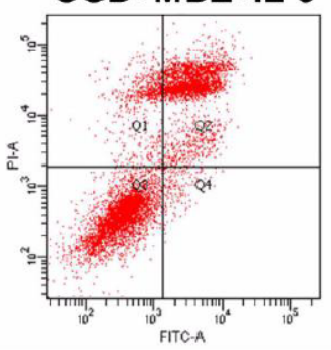

B

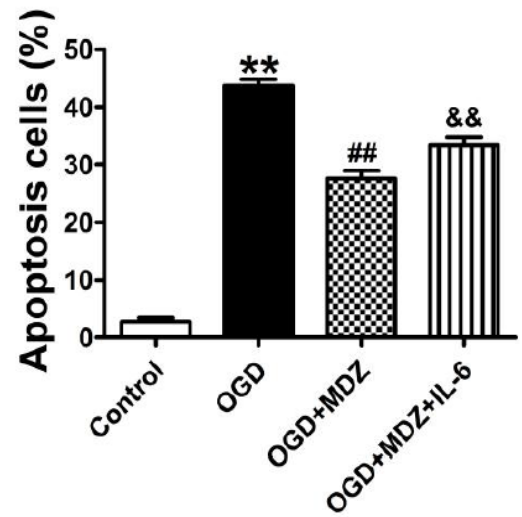

Fig. 3. The apoptotic rate of astrocytes was detected by FITC-annexin V labeling and flow cytometric analysis. Astrocytes were pre-treated with midazolam and co-treated with or without IL-6 under OGD condition. Note that midazolam significantly inhibited the apoptotic rate of astrocytes under OGD condition, and IL- 6 could attenuate the anti-apoptotic role of midazolam. The data are presented as the mean \pm SEM for three independent experiments. ${ }^{* *} \mathrm{P}<0.01$ vs control, ${ }^{*} \mathrm{P}<0.05$ vs $\mathrm{OGD}$ group, ${ }^{\&} \mathrm{P}<0.01$ vs $\mathrm{OGD}+\mathrm{MDZ}$ group.

A

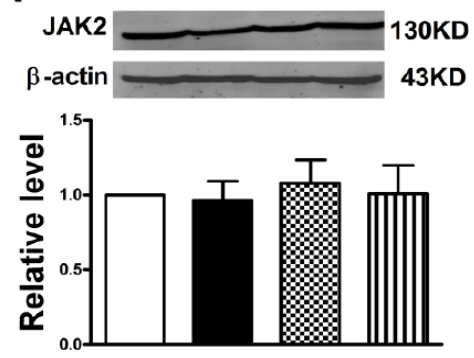

C
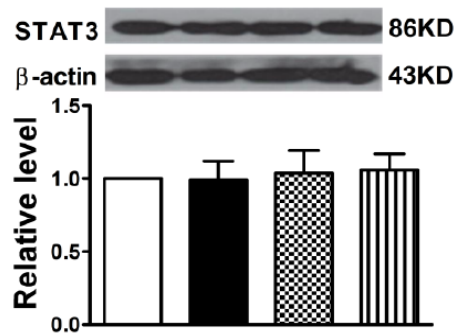

B
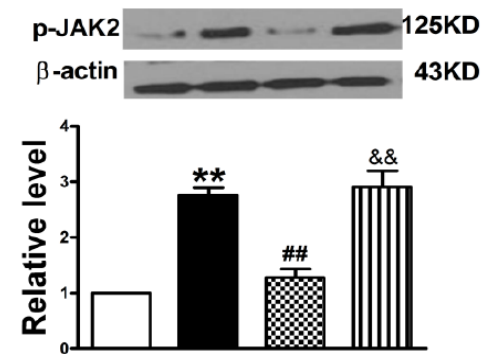

D

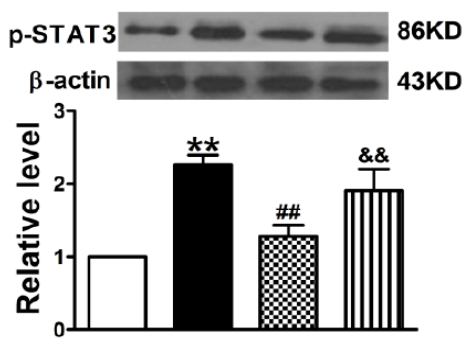

Fig. 4. Midazolam inhibits the JAK2/STAT3 cell signal proteins expression in astrocytes. Quantitative analysis of JAK2, p-JAK2, STAT3, and p-STAT3 expression in different treatment groups were examined by Western blotting. $\beta$-actin was used as an internal control. Data are mean $\pm \mathrm{SEM}, \mathrm{n}=3,{ }^{* *} \mathrm{P}<0.01$ vs control, $" \mathrm{P}<0.05$ vs OGD group, ${ }^{\&} \mathrm{P}<0.01$ vs $\mathrm{OGD}+\mathrm{MDZ}$ group.

played an anti-apoptotic role in astrocytes under OGD condition, supporting the notion that midazolam has a therapeutic potential in ischemic brain injury. 


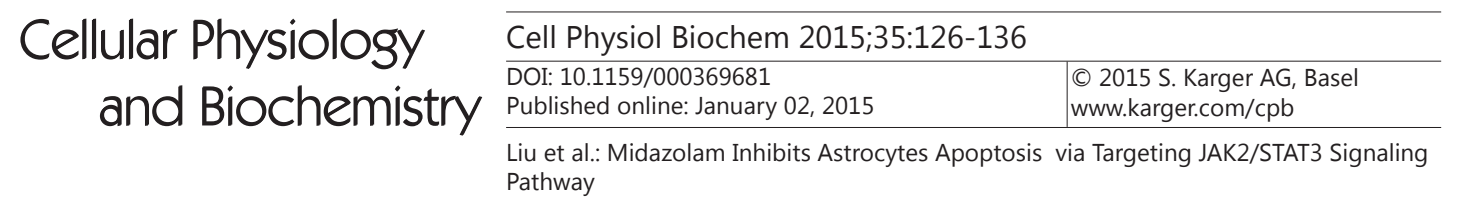

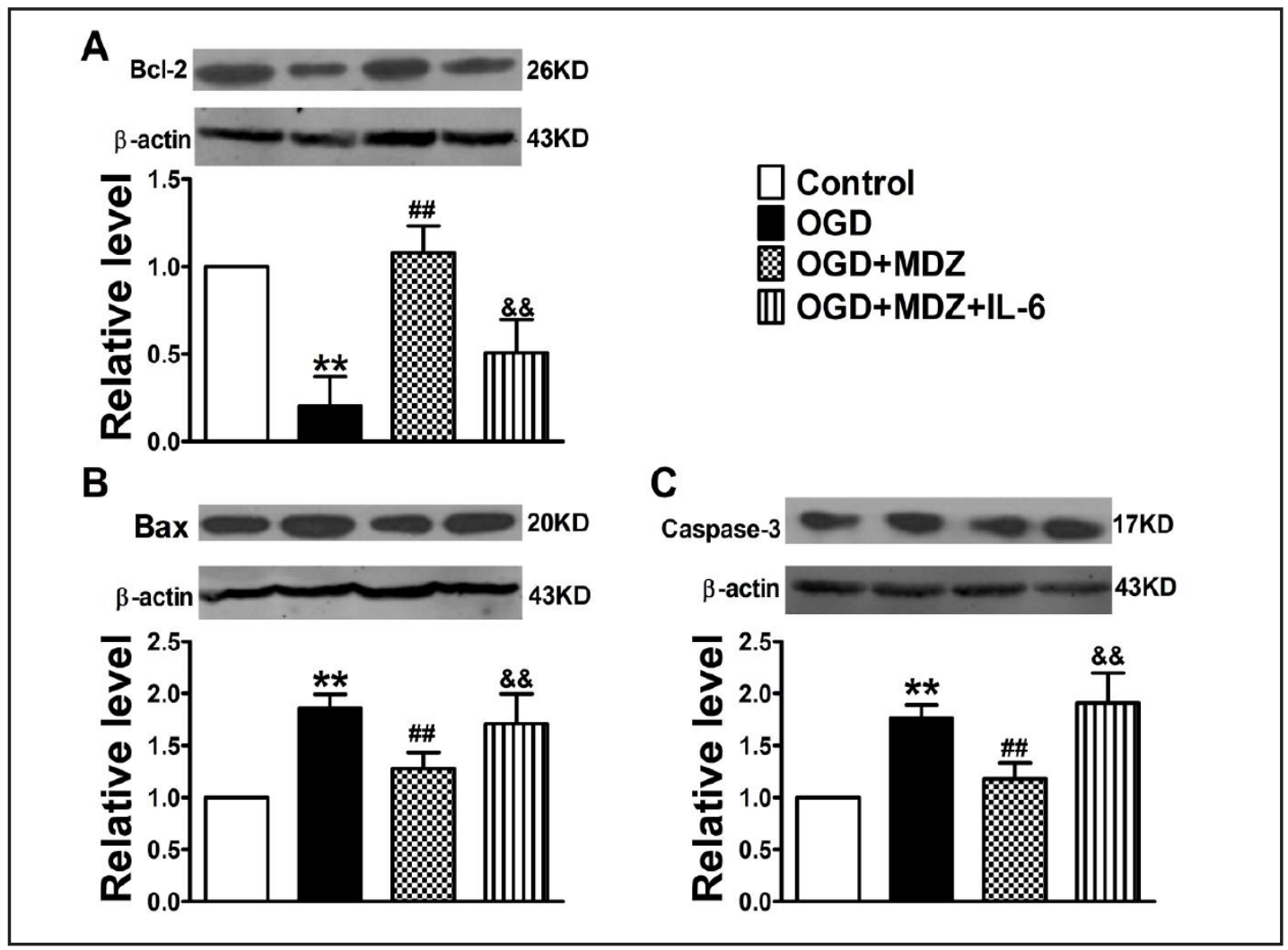

Fig. 5. Midazolam influences the expression of the apoptosis-related proteins Bcl-2, Bax and Caspase-3 in astrocytes. Quantitative analysis of Bcl-2, Bax and Caspase-3 expression in astrocytes in different treatment groups by western blotting. $\beta$-actin was used as an internal control. Data are mean $\pm S E M$, $\mathrm{n}=3,{ }^{* *} \mathrm{P}<0.01$ vs control, ${ }^{\#} \mathrm{P}<0.05$ vs OGD group, ${ }^{\& \&} \mathrm{P}<0.01$ vs $0 \mathrm{GD}+\mathrm{MDZ}$ group.

Midazolam inhibits astrocytes apoptosis under OGD condition through targeting JAK2/ STAT3 signaling pathway

Previous study has experimentally identified that JAK2/STAT3 signal pathway was involved in regulating cell apoptosis [26, 27]. In this study, we firstly confirmed midazolam played an anti-apoptotic role via the regulation of JAK2/STAT3 signal pathway in astrocytes under OGD condition (Figure 4A-4D).We found that, under OGD condition, p-JAK2 and p-STAT3 proteins expression in astrocytes markedly increased, while midazolam significantly inhibited the p-JAK2 and p-STAT3 proteins expression in astrocytes (Fig. 4A-4D). In addition, IL- 6 could attenuate the effects of midazolam, and in OGD+MDZ+IL- 6 group, the p-JAK2 and p-STAT3 proteins expression in astrocytes significantly increased compared with OGD+MDZ group (Fig. 4A-4D).The above results revealed that midazolam targeted JAK2/STAT3 signaling pathway and then inhibited apoptosis in astrocytes under OGD condition.

Midazolam regulates the apoptotic proteins expression in astrocytes under OGD condition

We then turned to investigate if midazolam could regulate the expression of JAK2/ STAT3 downstream apoptotic proteins in astrocytes under OGD condition. Western blotting analysis showed that midazolam could up-regulate the expression of anti-apoptotic protein Bcl-2 and down-regulate the expression of Bax and Caspase- 3 in astrocytes under OGD condition relative to OGD group (Fig. 5). Concomitantly, compared with OGD group, the production of Bax and Caspase-3 was decreased in midazolam pre-treatment group (Fig. 5). However, the IL- 6 could abrogate the anti-apoptotic effects of midazolam in astrocytes under OGD condition (Fig. 5). 


\section{Discussion}

The present study yielded several novel findings. First, we demonstrated, for the first time, that midazolam has protective effects on the proliferation and apoptosis of astrocytes under OGD condition. Secondly, we found that midazolam inhibits OGD-induced apoptosis, possibly partially by inhibiting the JAK2/STAT3 signaling pathway in brain astrocytes. Third, midazolam produce protective effects in astrocytes under OGD condition, which may be related to regulating apoptosis-related protein expression. In brief, these findings not only help us understand the mechanisms underlying the ischemic protective effects of midazolam but also advance our view of midazolam that may serve as potential drug in ischemic brain injury.

Brain ischemia injury is a subject of intense focus in basic and clinical research, which is involved in complicated pathophysiological processes. A growing of evidence shows that many cellular and molecular signaling pathways modulate neuronal homeostasis in the ischemic brain. Signaling transduction molecules such as protein kinase B and C are involved in neuroprotection by modulating apoptotic factors such as p53, B cell lymphoma2 -associated death promoter (BAD), and caspase-3 [28]. Furthermore, the astrocytetargeted overexpression of superoxide dismutase 2 or heat shock protein 72 significantly reduces the loss of CA1 hippocampal neurons in a forebrain ischemia model [29]. In a recent study, Soledad Calvo et al. found that inhibition of selenoprotein S1 by small interfering RNA severely increases astrocyte injury caused by OGD, suggesting that selenoprotein S protects astrocytes against ischemia [30]. Connexin43, a principal gap junction protein of astrocytes, is involved in protection from ischemic injury. Knockdown of connexin43 in mice astrocytes showed a markedly increased infarct volume and amplified inflammatory response and cell apoptosis [31]. STATs are a family of transcription factors involved in many cellular activities [32]. It has been reported that STAT3 activation was found in numerous cell types in the brain including neurons, astrocytes, microglia, endothelial cells, monocytes and macrophages, involving in neuronal development, neuroprotection, and regeneration after ischemic injury [33-35]. Perez-Pinzon et al. have demonstrated phosphorylation and nuclear translocation of STAT3 in response to preconditioning by OGD in mixed cortical-glial co-cultures [36]. In addition, activation of STAT3 by ischemia or brain injury could regulate the apoptosisrelated proteins $\mathrm{Bcl}-2$ and Bax expression resulting in anti-apoptotic events in the cells [37]. As JAK2 is up-stream to STAT3, we assumed that blocking JAK2 phosphorylation prevents STAT3 phosphorylation, leading to decreased downstream biological consequences of STAT3 activation. In agreement with hypothesis, data from current study disclosed that midazolam could block JAK2 phosphorylation, and then prevented STAT3 phosphorylation. We further disclosed that the prevention of ischemic-induced JAK2 and the down-stream STAT3 phosphorylation with midazolam could alleviate astrocytes apoptosis induced by OGD. In addition, we co-treated astrocytes with JAK phosphorylation activator IL-6, which abrogated the role of midazolam and increased both JAK2 and STAT3 phosphorylation in astrocytes. These changes may be a novel molecular and cellular mechanism underlying the ischemic neuroprotective effects of the midazolam.

Ischemia is the most common cause of brain injury. Astrocytes are essential for neuronal survival and function recovery, neural repair, and neurogenesis, especially in ischemic brain. However, recent studies have less focused on brain astrocytes, and relatively more emphasis on neurons during cerebral ischemia-reperfusion injury. Astrocytes are involved in maintaining brain integrity in conditions of cerebral ischemia-reperfusion injury. And astrocyte dysfunction during cerebral ischemia may damage the survival ability of neurons [38]. Thus, it is possible that interventions aimed at blocking astrocyte apoptosis could both limit neuron death and promote recovery. Midazolam, a benzodiazepine, is commonly used for anesthesia and sedation in the intensive care unit and the operating room where there is a risk of cerebral ischemia. Recent researches mostly focused on the effects of midazolam on neuroprotection after transient focal cerebral ischemia [39]. However, till now, the cytoprotective effect of midazolam on brain astrocytes has not been clarified in detail. In

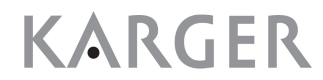




\section{Cellular Physiology Cell Physiol Biochem 2015;35:126-136

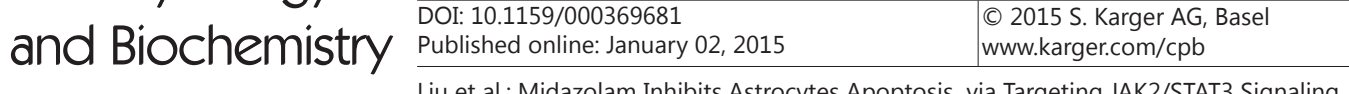 \\ Liu et al.: Midazolam Inhibits Astrocytes Apoptosis via Targeting JAK2/STAT3 Signaling Pathway}

order to investigate the possible cytoprotective effect of midazolam on OGD-induced damages in astrocytes, the cells were pretreated with $1,3,10,15$ or $30 \mu \mathrm{M}$ midazolam for $2 \mathrm{~h}$ and then cultured under OGD condition for $6 \mathrm{~h}$. Interestingly, in this study, we found that pretreatment with midazolam reduced OGD-induced cell death of astrocytes in a dose dependent manner, with a remarkable cytoprotective effect at $10 \mu \mathrm{M}$ midazolam. However, the cell survival went down when the dose went up to $15 \mu \mathrm{M}$. So, the expression level of JAK2 or p-JAK2 in astrocytes might be midazolam dose dependent in this study, which could explain cell viability went down while the midazolam dose went up to $15 \mu \mathrm{M}$ instead. In this current study, we firstly demonstrated that midazolam could inhibit apoptosis of brain astrocytes cultured under oxygen glucose deprivation condition via targeting. However, in another study, Gaza et al. have demonstrated that midazolam could induce human lymphoma and neuroblastoma cell lines apoptosis via activation of the mitochondrial in a concentrationdependent manner [40]. Thus, there are numerous mechanisms by which midazolam can potentially act to protect the brain against ischemic injury or to induce human lymphoma and neuroblastoma cell lines apoptosis. In brief, it should be pointed out that our studies were performed in vitro and the findings may not be extrapolated directly to humans. And the results of this study call for a further precaution applying to animals in vivo and patients with cerebral ischemia. Nevertheless, the findings open the door for further researches to disclose whether the anti-apoptotic role of midazolam in brain astrocytes also operate in the clinical setting.

Taken together, in this study we demonstrated that midazolam played an important role in promoting proliferation and inhibiting apoptosis of brain astrocytes cultured under oxygen glucose deprivation condition. In vitro experiment results showed that midazolam is able to alleviate cell apoptosis in brain astrocytes and the underlying mechanism involves an inhibition of JAK2/STAT3 signal pathway. This study indicates that application of midazolam may be a promising intervention in the management of brain astrocytes and the associated pathological processes.

\section{Disclosure Statement}

No potential conflicts of interest were disclosed.

\section{Acknowledgements}

This work was supported, in part, by the Wu Jieping Special Fund (320.6250. 12262), Heilongjiang Provincial Natural Science Foundation (D201071), the Third Hospital Fund (JJZ2011-09), the National Natural Science Foundation of China (81401457) and the Heilongjiang Health and Family Planning Commission (2014-385).

\section{Reference}

1 Grabowska-Fudala B, Jaracz K, Gorna K: stroke incidence, case fatality and mortality--current trends and future prognosis. Przegl Epidemiol 2010;64:439-442.

-2 Sypecka J, Sarnowska A, Gadomska-Szablowska I, Lukomska B, Domanska-Janik K: Differentiation of glia-committed ng2 cells: The role of factors released from hippocampus and spinal cord. Acta Neurobiol Exp(Wars) 2013;73:116-129.

3 Pizzurro DM, Dao K, Costa LG: Diazinon and diazoxon impair the ability of astrocytes to foster neurite outgrowth in primary hippocampal neurons. Toxicol Appl Pharmacol 2014;274:372-382. 


\section{Cellular Physiology $\quad$ Cell Physiol Biochem 2015;35:126-136 and Biochemistry \begin{tabular}{l|l}
$\begin{array}{l}\text { DOI: 10.1159/000369681 } \\
\text { Published online: January 02, } 2015\end{array}$ & $\begin{array}{l}\text { O 2015 S. Karger AG, Basel } \\
\text { www.karger.com/cpb }\end{array}$ \\
\cline { 1 - 2 }
\end{tabular} \\ Liu et al.: Midazolam Inhibits Astrocytes Apoptosis via Targeting JAK2/STAT3 Signaling Pathway}

4 Lee JC, Ahn JH, Kim IH, Park JH, Yan BC, Cho GS, Ohk TG, Park CW, Cho JH, Kim YM, Lee HY, Won MH: Transient ischemia-induced change of ccr7 immunoreactivity in neurons and its new expression in astrocytes in the gerbil hippocampus. J Neurol Sci 2014;336:203-210.

5 Scheiber IF, Dringen R: Astrocyte functions in the copper homeostasis of the brain. Neurochem Int 2013;62:556-565.

-6 Kimelberg HK: Supportive or information-processing functions of the mature protoplasmic astrocyte in the mammalian cns? A critical appraisal. Neuron Glia Biol 2007;3:181-189.

-7 Anderson CM, Swanson RA: Astrocyte glutamate transport: Review of properties, regulation, and physiological functions. Glia 2000;32:1-14.

-8 Fang Q Hu WW, Wang XF, Yang Y, Lou GD, Jin MM, Yan HJ, Zeng WZ, Shen Y, Zhang SH, Xu TL, Chen Z: Histamine up-regulates astrocytic glutamate transporter 1 and protects neurons against ischemic injury. Neuropharmacology 2014;77:156-166.

-9 Oliva I, Fernandez M, Martin ED: Dopamine release regulation by astrocytes during cerebral ischemia. Neurobiol Dis 2013;58:231-241.

10 Kalay S, Oztekin O, Tezel G, Aldemir H, Sahin E, Koksoy S, Akcakus M, Oygur N: Role of immunoglobulin in neuronal apoptosis in a neonatal rat model of hypoxic ischemic brain injury. Exp Ther Med 2014;7:734738.

11 Song J, Park KA, Lee WT, Lee JE: Apoptosis signal regulating kinase 1 (ask1): Potential as a therapeutic target for alzheimer's disease. Int J Mol Sci 2014;15:2119-2129.

12 Naziroglu M, Kutluhan S, Ovey IS, Aykur M, Yurekli VA: Modulation of oxidative stress, apoptosis, and calcium entry in leukocytes of patients with multiple sclerosis by hypericum perforatum. Nutr Neurosci 2014;17:214-221.

13 Chou WW, Wang YS, Chen KC, Wu JM, Liang CL, Juo SH: Tannic acid suppresses ultraviolet b-induced inflammatory signaling and complement factor b on human retinal pigment epithelial cells. Cell Immunol 2012;273:79-84.

14 Xie X, Chan KS, Cao F, Huang M, Li Z, Lee A, Weissman IL, Wu JC: Imaging of stat3 signaling pathway during mouse embryonic stem cell differentiation. Stem cells Dev 2009;18:205-214.

15 Chen M, Liu Y, Yi D, Wei L, Li Y, Zhang L: Tanshinone iia promotes pulmonary artery smooth muscle cell apoptosis in vitro by inhibiting the jak2/stat3 signaling pathway. Cell Physiol Biochem 2014;33:11301138.

-16 Erta M, Quintana A, Hidalgo J: Interleukin-6, a major cytokine in the central nervous system. Int J Biol Sci 2012;8:1254-1266.

17 Ohno M, Kohyama J, Namihira M, Sanosaka T, Takahashi JA, Hashimoto N, Nakashima K: Neuropoietin induces neuroepithelial cells to differentiate into astrocytes via activation of stat3. Cytokine 2006;36:1722.

18 Oh HM, Lee SW, Yun BR, Hwang BS, Kim SN, Park CS, Jeoung SH, Kim HK, Lee WS, Rho MC: Vigna angularis inhibits il-6-induced cellular signalling and ameliorates collagen-induced arthritis. Rheumatology (Oxford) 2014;53:56-64.

19 Trilling M, Le VT, Rashidi-Alavijeh J, Katschinski B, Scheller J, Rose-John S, Androsiac GE, Jonjic S, Poli V, Pfeffer K, Hengel H: "Activated" stat proteins: A paradoxical consequence of inhibited jak-stat signaling in cytomegalovirus-infected cells. J Immunol 2014;192:447-458.

20 Shinday NM, Sawyer EK, Fischer BD, Platt DM, Licata SC, Atack JR, Dawson GR, Reynolds DS, Rowlett JK: Reinforcing effects of compounds lacking intrinsic efficacy at alpha1 subunit-containing gabaa receptor subtypes in midazolam- but not cocaine-experienced rhesus monkeys. Neuropsychopharmacology 2013;38:1006-1014.

21 Tanabe K, Kozawa O, Iida H: Midazolam suppresses interleukin-1beta-induced interleukin-6 release from rat glial cells. J Neuroinflammation 2011;8:68.

22 Bentz K, Molcanyi M, Hess S, Schneider A, Hescheler J, Neugebauer E, Schaefer U: Neural differentiation of embryonic stem cells is induced by signalling from non-neural niche cells. Cell Physiol Biochem 2006;18:275-286.

23 Zhang W, Qian CY, Li SQ: Protective effect of sgk1 in rat hippocampal neurons subjected to ischemia reperfusion. Cell Physiol Biochem 2014;34:299-312. 


\section{Cellular Physiology $\quad$ Cell Physiol Biochem 2015;35:126-136 \begin{tabular}{l|l|l} 
and Bioch 10.1159/000369681 & $\begin{array}{l}\text { O 2015 S. Karger AG, Basel } \\
\text { www.karger.com/cpb }\end{array}$ \\
\hline
\end{tabular} \\ Liu et al.: Midazolam Inhibits Astrocytes Apoptosis via Targeting JAK2/STAT3 Signaling Pathway}

24 Li M, Liu X, Yue H, Xiong W, Gu J, Xu M: Transplantation of n -acetyl aspartyl-glutamate synthetase-activated neural stem cells after experimental traumatic brain injury significantly improves neurological recovery. Cell Physiol Biochem 2013;32:1776-1789.

25 Xu H, Liu X, Zhao J: Down-regulation of mir-3928 promoted osteosarcoma growth. Cell Physiol Biochem 2014;33:1547-1556.

26 Ge G, Zhang Q Ma J, Qiao Z, Huang J, Cheng W, Wang H: Protective effect of salvia miltiorrhiza aqueous extract on myocardium oxidative injury in ischemic-reperfusion rats. Gene 2014;546:97-103.

-27 Zhang Z, Wu Z, Zhu X, Hui X, Pan J, Xu Y: Hydroxy-safflor yellow a inhibits neuroinflammation mediated by abeta(1)(-)(4)(2) in bv-2 cells. Neurosci lett 2014;562:39-44.

-28 Takita H, Yoneya S, Gehlbach PL, Wei LL, Mori K: An empty e1, e3, e4 adenovirus vector protects photoreceptors from light-induced degeneration. J Ocul Biol Dis Infor 2008;1:30-36.

29 Xu L, Emery JF, Ouyang YB, Voloboueva LA, Giffard RG: Astrocyte targeted overexpression of hsp72 or sod2 reduces neuronal vulnerability to forebrain ischemia. Glia 2010;58:1042-1049.

- 30 Fradejas N, Pastor MD, Mora-Lee S, Tranque P, Calvo S: Seps1 gene is activated during astrocyte ischemia and shows prominent antiapoptotic effects. J Mol Neurosci: MN 2008;35:259-265.

- 31 Froger N, Orellana JA, Calvo CF, Amigou E, Kozoriz MG, Naus CC, Saez JC, Giaume C: Inhibition of cytokineinduced connexin43 hemichannel activity in astrocytes is neuroprotective. Mol Cell Neurosci 2010;45:3746.

-32 O'Shea JJ, Holland SM, Staudt LM: Jaks and stats in immunity, immunodeficiency, and cancer. N Engl J Med 2013;368:161-170.

33 Yu L, Chen C, Wang LF, Kuang X, Liu K, Zhang H, Du JR: Neuroprotective effect of kaempferol glycosides against brain injury and neuroinflammation by inhibiting the activation of nf-kappab and stat3 in transient focal stroke. PloS One 2013;8:e55839.

-34 Wei ZZ, Yu SP, Lee JH, Chen D, Taylor TM, Deveau TC, Yu AC, Wei L: Regulatory role of the jnk-stat1/3 signaling in neuronal differentiation of cultured mouse embryonic stem cells. Cell Mol Neurobiol 2014;34:881-893.

-35 Leibinger M, Andreadaki A, Diekmann H, Fischer D: Neuronal stat3 activation is essential for cntf- and inflammatory stimulation-induced cns axon regeneration. Cell Death Dis 2013;4:e805.

-36 Kim EJ, Raval AP, Perez-Pinzon MA: Preconditioning mediated by sublethal oxygen-glucose deprivationinduced cyclooxygenase-2 expression via the signal transducers and activators of transcription 3 phosphorylation. J Cereb Blood Flow Metab 2008;28:1329-1340.

- 37 Zhang F, Wang S, Cao G, Gao Y, Chen J: Signal transducers and activators of transcription 5 contributes to erythropoietin-mediated neuroprotection against hippocampal neuronal death after transient global cerebral ischemia. Neurobiol Dis 2007;25:45-53.

- 38 Ashpole NM, Chawla AR, Martin MP, Brustovetsky T, Brustovetsky N, Hudmon A: Loss of calcium/ calmodulin-dependent protein kinase ii activity in cortical astrocytes decreases glutamate uptake and induces neurotoxic release of atp. J Biol Chem 2013;288:14599-14611.

-39 Harman F, Hasturk AE, Yaman M, Arca T, Kilinc K, Sargon MF, Kaptanoglu E: Neuroprotective effects of propofol, thiopental, etomidate, and midazolam in fetal rat brain in ischemia-reperfusion model. Childs Nerv Syst 2012;28:1055-1062.

-40 Stevens MF, Werdehausen R, Gaza N, Hermanns H, Kremer D, Bauer I, Kury P, Hollmann MW, Braun S: Midazolam activates the intrinsic pathway of apoptosis independent of benzodiazepine and death receptor signaling. Reg Anesth Pain Med 2011;36:343-349. 